ORIGINAL ARTICLE

\title{
IHC for Her2 with CBE356 antibody is a more accurate predictor of Her2 gene amplification by FISH than HercepTest $^{\mathrm{TM}}$ in breast carcinoma
}

\author{
R Ainsworth, J M S Bartlett, J J Going, E A Mallon, A Forsyth, J Richmond, W Angerson, A Watters, \\ B Dunne
}

J Clin Pathol 2005;58:1086-1090. doi: 10.1136/jcp.2004.021576

See end of article for authors' affiliations

.....................

Correspondence to:

Dr B Dunne, Department of Pathology, Central Pathology Laboratory, St James's Hospital, Dublin 8, Ireland; barbaradunne@ hotmail.com

Accepted 15 February 2005

\begin{abstract}
Background: Her2 (c-erbB-2/neu) overexpression in breast carcinoma predicts response to the anti-Her2 monoclonal antibody, trastuzumab, and is associated with a poor prognosis. When considering patients for trastuzumab treatment, Her2 protein expression is measured by imunohistochemistry $(\mathrm{IHC})$ and, where staining is equivocal, by fluorescence in situ hybridisation (FISH) detection of Her2 gene amplification. Aims: To compare $\mathrm{IHC}$ using CBE356 with $\mathrm{IHC}$ using the Food and Drug Administration approved HercepTest ${ }^{\mathrm{TM}}$.

Methods: CBE356 and HercepTest were analysed using 167 FISH characterised breast carcinomas. Immunohistochemical expression of Her2 was measured semiquantitatively. Sensitivity, specificity, predictive values, and overall accuracy were calculated for both $\mathrm{IHC}$ methods using gene amplification by FISH as the end point, and IHC and FISH assays were tested in Kaplan-Meier survival analysis.

Results: The accuracy, sensitivity, specificity, positive predictive value (PPV), and negative predictive value (NPV) of CBE356 positive (2+ and 3+) cases were $94 \%, 89 \%, 95 \%, 84 \%$, and $97 \%$, respectively, and of HercepTest positive (2+ and $3+$ ) cases were $91 \%, 66 \%, 98 \%, 92 \%$, and $91 \%$, respectively. A positive result with CBE356, HercepTest, or FISH was associated with significantly decreased overall survival (log rank $p=0.005, p=0.0017$, and $p=0.0005$, respectively).

Conclusions: Positive IHC staining for Her2 using CBE356 is 3\% more accurate and $23 \%$ more sensitive at predicting Her2 gene amplification by FISH than positive staining with HercepTest. Negative IHC using CBE356 antibody is $6 \%$ more likely to represent a truly negative result than negative staining with HercepTest. Overall, CBE356 was a more accurate predictor of Her2 gene amplification by FISH than HercepTest.
\end{abstract}

\begin{abstract}
"Accurate testing for Her2 aims to ensure that costly and potentially toxic trastuzumab treatment is not given to patients with non-overexpessing tumours and that every patient who might benefit from trastuzumab receives appropriate treatment"
\end{abstract}

Determination of Her2 overexpression in breast carcinoma is now of major clinical importance with the advent of the humanised anti-Her2 monoclonal antibody trastuzumab, which is beneficial in patients with metastatic breast carcinoma. ${ }^{17}{ }^{18}$ Demonstration of Her2 overexpression is a prerequisite for treatment with trastuzumab because it acts by specifically targeting Her2 overexpressing carcinoma cells. Accurate testing for Her2 aims to ensure that costly and potentially toxic trastuzumab treatment is not given to patients with non-overexpessing tumours and that every patient who might benefit from trastuzumab receives appropriate treatment.

Immunohistochemistry (IHC) and fluorescence in situ hybridisation (FISH) are established methods for detecting
Her2 overexpression in formalin fixed, paraffin wax embedded tissue in diagnostic laboratories. IHC for Her2 is a robust screening test when supported by rigorous quality control measures, ${ }^{19}$ and has a continuing role in diagnostic Her2 testing. There is a wide array of IHC antibodies with varying sensitivities available for Her2 testing, but the Food and Drug Administration (FDA) approved HercepTest IHC kit has achieved wide usage. The use of FISH to detect Her2 gene amplification in diagnostic laboratories is usually reserved for cases with equivocal staining by IHC. The FDA approved PathVysion FISH kit is one of the most widely used FISH assay systems.

All diagnostic laboratories will require access to accurate, reproducible Her2 testing and part of that process will be to put in place tests that are financially viable and have been validated against FDA approved tests and against survival. Our aim was to test the accuracy of IHC staining with the CBE356 antibody for the detection of Her2 overexpression in a series of breast cancers. We compared the sensitivity and specificity and overall accuracy of this antibody with HercepTest IHC at predicting gene amplification by PathVysion FISH and we examined the prognostic value of each test in terms of overall survival.

\footnotetext{
Abbreviations: $\mathrm{Cl}$, confidence interval; $\mathrm{FDA}$, Food and Drug Administration; FISH, fluorescence in situ hybridisation; IHC, immunohistochemistry
} 


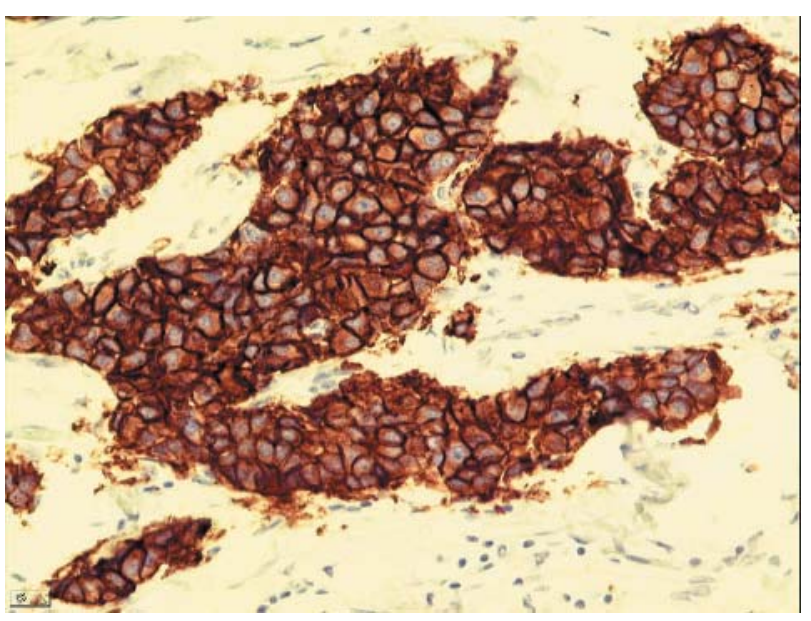

Figure 1 Her2 immunohistochemistry using CBE356 showing 3+ staining; original magnification, $\times 200$.

\section{MATERIALS AND METHODS Tissue samples}

Formalin fixed, paraffin wax embedded blocks of 167 breast carcinomas, resected between 1984 and 1998, which had prospective follow up, were retrieved from the files of the Glasgow Royal Infirmary, UK. All patients were female and the mean age was 70 years (range, 30-87). There were 152 invasive ductal carcinomas, 12 invasive lobular carcinomas, two medullary carcinomas, and one primary squamous cell carcinoma. Twenty two tumours were grade 1, 63 were grade 2 , and 82 were grade 3 . The mean tumour size was $34 \mathrm{~mm}$ (range, 6-130). Seventy six tumours were axillary lymph node positive, 68 were node negative, and 23 cases did not have axillary lymph node dissection.

\section{Immunohistochemistry HercepTest}

IHC using HercepTest ${ }^{\mathrm{TM}}$ (anti-Her2 polyclonal antibody; Dako, Ely, UK) on a Techmate automated staining system (Dako) was performed on 165 cases according to the kit instructions, with epitope retrieval in $10 \mathrm{mmol} /$ litre citrate buffer in a water bath at $95^{\circ} \mathrm{C}$ for 40 minutes. Semiquantitative scoring (by JJG) using the standard protocol (with reference to images published by Dako) was

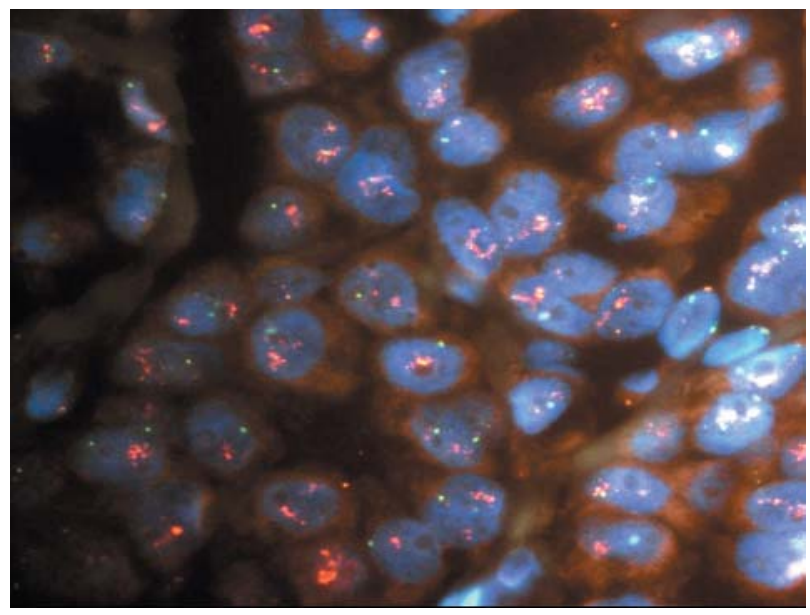

Figure 2 PathVysion fluorescence in situ hybridisation showing Her2 gene amplification as a red signal. The green signal represents chromosome 17. performed on all cases where scores of $0,1+, 2+$, and $3+$ were allocated if there was no staining or if $10 \%$ or more of carcinoma cells showed weak, moderate, and intense membrane staining, respectively.

\section{CBE356}

IHC was performed on all 167 cases on a Techmate automated staining system (Dako) using CBE356 mouse monoclonal antibody, clone 10A7 (Novocastra, Newcastle Upon Tyne, UK ) to the external domain of Her2. The primary antibody concentration and antigen retrieval methods were first calibrated against FISH characterised tumours using dilutions of $1 / 40,1 / 80$, and $1 / 200$, with and without microwave pressure cooker antigen retrieval. A 1/200 primary antibody dilution (60 minutes of incubation with primary antibody at $25^{\circ} \mathrm{C}$ ) was shown to be the most sensitive and specific, with antigen retrieval in a microwave pressure cooker for 12 minutes in EDTA buffer. Semiquantitative scoring (by BD) using the standard protocol (with reference to images published by Dako) was performed on all cases where scores of $0,1+, 2+$, and $3+$ were allocated if there was no staining or if $10 \%$ or more of carcinoma cells showed weak, moderate, and intense membrane staining, respectively.

\section{$\mathrm{FISH}$}

FISH analysis using a PathVysion detection kit was performed on all 167 cases after pretreatment with the VP2000 tissue processor (Vysis UK Ltd, Richmond, UK). Hybridisation was performed on a Misha hybridisation station (Shandon, Runcorn, Cheshire, UK). After posthybridisation washes, sealed slides mounted in $0.5 \mu \mathrm{g} / \mathrm{ml}$ DAPI in "Vectashield" were analysed by epifluorescence microscopy. FISH stained sections were scanned at $\times 1000$ magnification and three separate carcinoma areas were identified. Twenty nuclei were assessed in each area, the chromosome 17 copy number was counted for each cell, and the ratio of Her2 signals to chromosome 17 signals was calculated. The normal mean Her2 to chromosome 17 ratio was defined as less than two, and a ratio greater than two was interpreted as gene amplification.

\section{Statistics}

Statistical analysis was performed using SSPS statistical package (version 6.1.3). Using FISH data as the end point, overall accuracy, sensitivity, specificity, and positive and negative predictive values were calculated for CBE356 positive staining $(2+$ and $3+$ cases combined) and for HercepTest positive staining (2+ and $3+$ cases combined). Follow up information was available on 165 patients. Kaplan-Meier disease specific survival curves were calculated for FISH amplified and FISH non-amplified cases, for HercepTest 0/1+, 2+, and 3+ cases, and for CBE356 0/1+, 2+, and $3+$ cases; $95 \%$ confidence intervals were calculated for the median survival in each case. The log rank test was used to compare survival curves. Patients who died of other causes were censored.

\section{RESULTS}

Staining with the CBE356 antibody showed a clear membrane pattern (fig 1) characteristic of the Her2 protein. IHC scores for both antibodies are shown for both FISH amplified and non-amplified cases (table 1). The overall accuracy of the CBE356 antibody was greater than that of the HercepTest (94\% and 91\%, respectively) when PathVysion FISH amplification (fig 2) was taken as the end point (table 2). The CBE356 and HercepTest results were comparable in terms of overall specificity (95\% and $98 \%$, respectively; table 2 ), but the CBE356 antibody showed a much greater overall 
Table 1 Semiquantitative CBE356 and HercepTest IHC scores against amplification of Her2 gene by FISH

\begin{tabular}{|c|c|c|c|c|c|c|c|c|}
\hline & \multicolumn{4}{|c|}{ CBE356 IHC score } & \multicolumn{4}{|c|}{ HercepTest score } \\
\hline & 0 & $1+$ & $2+$ & $3+$ & 0 & $1+$ & $2+$ & $3+$ \\
\hline Her2 FISH not amplified & 107 & 18 & 4 & 2 & 125 & 3 & 1 & 1 \\
\hline Her2 FISH amplified & 2 & 2 & 6 & 26 & 9 & 3 & 12 & 11 \\
\hline Total & 109 & 20 & 10 & 28 & 134 & 6 & 13 & 12 \\
\hline$\%$ Of total & 65 & 12 & 6 & 17 & 81 & 4 & 8 & 7 \\
\hline
\end{tabular}

FISH, fluorescence in situ hybridisation; IHC, immunohistochemistry.

sensitivity than the HercepTest (89\% and $66 \%$ respectively) and a greater negative predictive value $(97 \%$ and $91 \%$, respectively), whereas the HercepTest showed a greater positive predictive value than CBE356 (92\% and 84\%, respectively).

\section{Survival analysis}

The maximum follow up was 17.2 years (median, 5; $\mathrm{n}=165)$. Patients without Her2 amplification survived significantly longer than those with amplification: median survival was 11.7 years (95\% confidence interval (CI), 7.4 to 15.9 ) and 2.6 years (95\% CI, 1.6 to 3.7), respectively (log rank: $p=0.0005$; fig 3 ). Patients with tumours scoring $0 / 1+$ with the CBE356 antibody survived significantly longer than those scoring $2+$ or $3+$, with a median survival of 11.7 years (95\% CI, 7.9 to 15.5 ), 2.9 years (95\% CI, 0 to 6.8 ), and 2.3 years (95\% CI, 0.3 to 4.3 ), respectively (log rank: $p=0.005$; fig 4). Similarly, survival was significantly longer in patients with tumours scoring 0 or $1+$ with the HercepTest than in those scoring $2+$ or $3+$, with a median survival of 11.7 years (95\% CI, 7.7 to 15.7 ), 2.3 years (95\% CI, 1.7 to 2.9 ), and 1.4 years (95\% CI, 0 to 5.8 ), respectively (log rank: $\mathrm{p}=0.0017$; fig 5).

\section{DISCUSSION}

There has been much debate recently regarding interlaboratory variability in Her2 testing that is centred predominantly on inconsistencies in IHC assays. Because selection of patients for trastuzumab treatment and for entry into trials investigating the benefit of this treatment in earlier disease is based on these assays, and because the cost of 12 weeks of monotherapy is at least $£ 5300$ /patient, accurate testing on formalin fixed, paraffin wax embedded tissue is of the utmost importance.

Inconsistencies in the sensitivity of IHC Her2 testing have been attributed to the use of different antibodies, differences in tissue fixation and IHC reagents, and the inclusion (or not) of antigen retrieval methods. ${ }^{20-23}$ The HercepTest kit has overcome some of these problems by using standardised methodology and reagents and by the inclusion of cell line controls. Nonetheless, the reported sensitivity of the HercepTest varies between different centres and, although it

Table 2 Comparison of CBE356 and HercepTest IHC positive cases according to sensitivity, specificity, accuracy and predictive values

\begin{tabular}{lll}
\hline & CBE356 2+/3+ & HT 2+/3+ \\
\hline Sensitivity & $89 \%$ & $66 \%$ \\
Specificity & $95 \%$ & $98 \%$ \\
PPV & $84 \%$ & $92 \%$ \\
NPV & $97 \%$ & $91 \%$ \\
Accuracy & $94 \%$ & $91 \%$ \\
\hline
\end{tabular}

$H T$, HercepTest; IHC, immunohistochemistry; NPV, negative predictive value; PPV, positive predictive value.

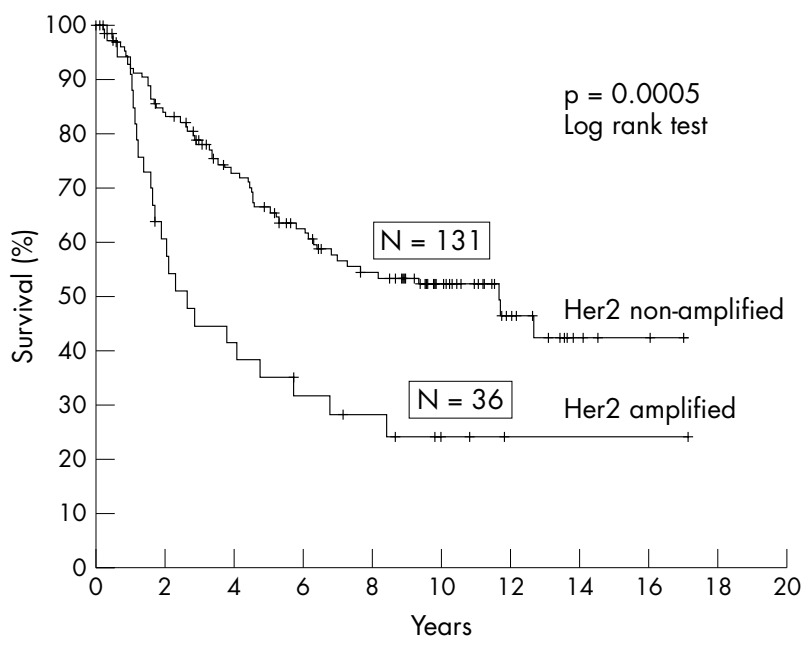

Figure 3 Kaplan-Meier survival curve showing the effect of Her2 amplification by fluorescence in situ hybridisation on disease specific survival. Vertical lines represent censored patients.

has achieved high sensitivity in some reports, ${ }^{24-28}$ it is costly and therefore its widespread use has budgetary implications for laboratories wishing to set up mandatory testing.

FISH is the gold standard for detecting Her2 amplification, ${ }^{29-31}$ but is dependent on adequately skilled personnel, and the assay is relatively expensive to set up and to perform when compared with in house immunohistochemical assays. Her2 amplification is usually associated with protein overexpression, ${ }^{12} 32$ and the current recommendation is to use

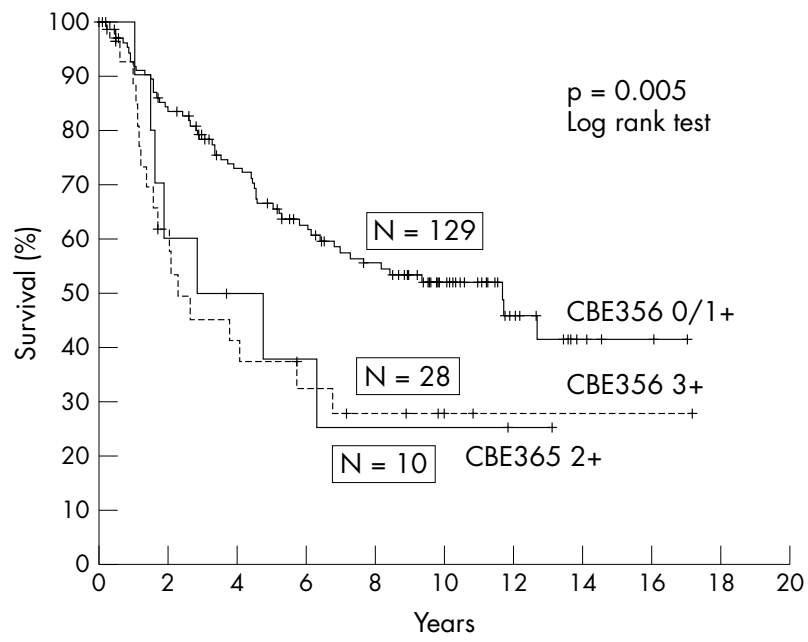

Figure 4 Kaplan-Meier survival curve showing the effect of CBE356 immunohistochemical staining for Her2 on disease specific survival. Vertical lines represent censored patients. 


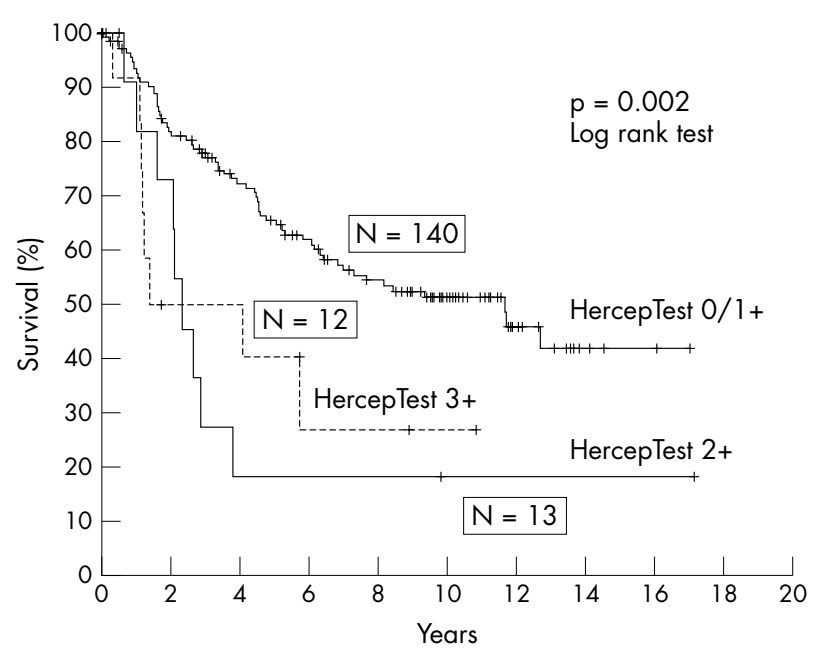

Figure 5 Kaplan-Meier survival curve showing the effect of HercepTest immunohistochemical staining on disease specific survival. Vertical lines represent censored patients.

IHC as a primary screening test and to select only cases that stain equivocally by IHC for FISH analysis. ${ }^{33-35}$

There is a wide variety of IHC antibodies for Her2 now available. We tested CBE356 and CB11 antibodies on a small pilot series of breast carcinomas and found CBE356 staining to be comparable to HercepTest staining in pattern and intensity. In our hands, background cytoplasmic staining was high with the CBIl antibody (Novocastra, Newcastle upon Tyne, UK), making interpretation difficult, so we did not include this antibody in the formal study. To optimise staining with CBE356, we calibrated the primary antibody concentration against amplification of Her2 with FISH in a training set before staining the large series. CBE356 has the advantage of being considerably less expensive than the HercepTest kit (costing less than $£ 5 /$ test and approximately $£ 30 /$ test, respectively). We tested the sensitivity and specificity of CBE356 IHC compared with PathVysion FISH analysis on a large series of breast carcinomas and we validated the IHC results in terms of survival.

In our study, positive IHC with CBE356 for Her2 was 89\% sensitive at predicting gene amplification by FISH, compared with a $66 \%$ sensitivity rate only using HercepTest IHC. Specificity using the two IHC methods is roughly similar, at $95 \%$ and $98 \%$, respectively (table 2 ). The main difference in sensitivity is accounted for by the fact that $35 \%$ of the total number of cases showing Her2 amplification by FISH had a score of zero or $1+$ using HercepTest, compared with only $12 \%$ with CBE356. According to the HercepTest manufacturer's

Take home messages

- We compared immunohistochemical (IHC) staining for Her2 using the CBE356 antibody with the HercepTest and found that CBE356 is 3\% more accurate and $23 \%$ more sensitive at predicting Her2 gene amplification by fluorescence in situ hybridisation (FISH) than positive staining with the HercepTest

- Negative IHC using the CBE356 antibody is $6 \%$ more likely to represent a truly negative result than negative staining with HercepTest

- Thus, CBE356 provides a cheaper, more accurate predictor of Her2 overexpression than the HercepTest guidelines, the expected proportions of $0 / 1+, 2+$, and $3+$ cases are $70 \%, 10 \%$, and $20 \%$, respectively. The negative rate using HercepTest in our series was $85 \%$, the $2+$ rate was $8 \%$, and the $3+$ rate was $7 \%$. The reason for the high number of $0 / 1+$ cases, apparently at the expense of the $3+$ cases, is not clear. One possible explanation is that the HercepTest assay had only recently been introduced to our laboratory at the time of staining, potentially resulting in technical or interpretative inexperience. The distribution of cases using CBE356 was closer to the guidelines, with a negative rate of $77 \%$, an equivocal rate of $6 \%$, and a positive rate of $17 \%$; $72 \%$ of the total amplified cases scored 3+ with CBE356 and 17\% scored $2+$ (table 1). Using the HercepTest, $34 \%$ of all amplified cases scored +2 and only $31 \%$ of cases scored +3 . Sixty percent of the CBE356 2+ cases were amplified, whereas almost all $(92 \%)$ of HercepTest $2+$ cases showed FISH amplification of Her2, explaining the higher positive predictive value of the HercepTest (92\%) compared with $84 \%$ for CBE356 (tables 1, 2 ). Other studies have shown a much lower level of FISH amplification of IHC $2+$ cases, ${ }^{19} 253637$ suggesting that a large proportion of the HercepTest $2+$ cases should have shown $3+$ staining. The increased likelihood of CBE356 rather than HercepTest IHC giving a 3+ staining pattern could reduce the number of cases requiring FISH analysis, at a cost of at least £80/test, representing a cost saving in addition to that afforded by the considerably cheaper CBE356 IHC assay.

\section{"CBE356 has the advantage of being considerably less expensive than the HercepTest kit (costing less than $£ 5 /$ test and approximately $£ 30 /$ test, respectively)"}

No other published study has tested the accuracy of CBE356 at predicting gene amplification. The $89 \%$ sensitivity of CBE356 coupled with 95\% specificity compares very favourably not only with the HercepTest in our series, but with other IHC studies. Press et al described the sensitivity of HercepTest to be $70 \%$ (based on cases showing moderate or high intensity staining) and that of CB1l to be $72 \%$ in a study of 117 molecularly characterised tumours, although their in house antibodies R60 (polyclonal) and 10H8 (monoclonal) were more sensitive at $91 \%$ and $88 \%$, respectively. ${ }^{23}$ Earlier reports using the HercepTest showed oversensitive staining compared with other antibodies, ${ }^{27}{ }^{28}$ but these results were based on expected rate of overexpression rather than comparison with gene amplification.

Therefore, we have shown that CBE356 IHC is both a more accurate and a more sensitive predictor of Her2 gene amplification by FISH than the FDA approved HercepTest in our series. Importantly, a negative result with CBE356 is more reassuring than a negative result with HercepTest. Truly positive cases are more likely to have strongly positive 3+ staining with CBE356 than with the HercepTest, reducing the need for FISH analysis. We have validated CBE356 IHC staining in terms of survival and shown that positive CBE356 IHC staining for Her2 is associated with a significantly shorter time to death from breast cancer $(p=0.005)$. As expected, patients with FISH amplification had a significantly shorter survival than those without amplification $(p=0.0005)$. For HercepTest staining, time to death from breast cancer was also significantly decreased in patients with $2+$ and $3+$ tumours compared with those whose tumours showed $0 / 1+$ staining $(p=0.0017)$.

In an era in which IHC is considered an effective screening tool for the detection of Her2 amplification in breast carcinoma, we propose that the use of CBE356 antibody should be considered by laboratories that wish to set up accurate and cost effective Her2 testing. Further studies with larger numbers are needed to corroborate our data, and 
further validation of IHC staining against response to trastuzumab treatment is required to confirm the robustness of this antibody.

\section{ACKNOWLEDGEMENTS}

The authors would like to thank NovoCastra Laboratories, UK for donating the primary antibody CBE356 for use in this study.

\section{Authors' affiliations}

R Ainsworth, J J Going, J Richmond, B Dunne, Department of Pathology, Glasgow Royal Infirmary, Glasgow G4 OSF, UK

J M S Bartlett, A Forsyth, W Angerson, A Watters, Endocrine Cancer Group, University of Glasgow G312ER, Glasgow, UK

E A Mallon, Department of Pathology, Western Infirmary, Glasgow G1 1 6NT, UK

\section{REFERENCES}

1 Slamen DJ, Clark GM, Wong SG, et al. Human breast cancer: correlation of relapse and survival with amplification of the Her2/neu oncogene. Science 1987; 235:177-82.

2 Rilke F, Colnaghi Ml, Cascinelli N, et al. Prognostic significance of Her2/neu expression in breast cancer and its relationship to other prognostic factors. Int J Cancer 1991:49:44-9.

3 Quenel N, Wafflart J, Bonichon F, et al. The prognostic value of c-erb B-2 in primary breast cancer: a study on 942 cases. Breast Cancer Res Treat 1995;35:283-91.

4 Press MF, Pike MC, Chazin VR, et al. HER2/neu expression in node negative breast cancers: direct tissue quantitation by computerized image analysis and association of overexpression with increased risk of recurrent disease. Cancer Res 1993;53:4960-70.

5 Press MF, Bernstein L, Thomas PA, et al. Her2/neu gene amplification by fluorescence in-situ hybridization: evaluation of archival specimens and utility as a marker of poor prognosis in node negative invasive breast carcinomas. $J$ Clin Oncol 1997;15:2894-904

6 Gullick WJ, Love SB, Wright C, et al. c-erb B-2 protein overexpression in breast cancer is a risk factor in patients with involved and uninvolved lymph nodes. Br J Cancer 1991;63:434-8.

7 Winstanley J, Cooke T, Murray GD, et al. The long term prognostic significance of c-erb B-2 in primary breast cancer. $\mathrm{Br} J$ Cancer 1991;63:447-50

8 Gusterson BA, Gelber RD, Goldhirsch A, et al. Prognostic importance of cerbB-2 expression in breast cancer: international (Ludwig) breast cancer study group. J Clin Oncol 1992;10:1049-56.

9 Andrulis IL, Bull SB, Blackstein ME, et al. neu/erbB-2 amplification identifies a poor prognosis group of women with node negative breast cancer. Toronto breast cancer study group. J Clin Oncol 1998;16:1340-9.

10 Muss HB, Thor AD, Berry DA, et al. cerbB-2 expression and response to adjuvant therapy in women with node positive early breast cancer. N Engl J Med 1994;330:1260-6

11 Tetu B, Brisson J, Plante V, et al. p53 and c-erbB-2 as markers of resistance to adjuvant chemotherapy in breast cancer. Mod Pathol 1998:11:823-30.

12 Thor AD, Berry DA, Budman DR, et al. erbB-2, p53 and efficacy of adjuvant therapy in lymph node-positive breast cancer. $J$ Natl Cancer Inst 1998;90:1346-60.

13 Paik S, Bryant J, Park C, et al. erbB-2 and response to doxorubicin in patients with axillary lymph node positive, hormone receptor negative breast cancer. J Natl Cancer Inst 1998;90:1361-70.

14 Wright C, Nicholson S, Angus B, et al. Relationship between c-erbB-2 protein product expression and response to endocrine therapy in advanced breast cancer. Br J Cancer 1992;65:118-21.

15 Carlomagno C, Perrone F, Gallo C, et al. c-erbB2 overexpression decreases the benefit of adjuvant tamoxifen in early-stage breast cancer without axillary lymph node metastases. J Clin Oncol 1996; 14:2702-8.
16 Siogren S, Inganas M, Lindgren A, et al. Prognostic and predictive value of cerbB2 overexpression in primary breast cancer, alone and in combination with other prognostic markers. J Clin Oncol 1998;16:462-9.

17 Goldenberg MM. Trastazumab, a recombinant DNA-derived humanized monoclonal antibody, a novel agent for the treatment of metastatic breas cancer. Clin Ther 1999;21:309-18.

18 Slamon DJ, Leyland-Jones B, Shak S, et al. Use of chemotherapy plus a monoclonal antibody against Her2 for metastatic breast cancer that overexpresses Her2. N Engl J Med 2001;344:783-92.

19 Dowsett M, Bartlett J, Ellis IO, et al. Correlation between immunohistochemistry (HercepTest) and fluorescence in situ hybridization (FISH) for Her-2 in 426 breast carcinomas from 37 centres. J Pathol 2003;199:418-23

20 Singleton TP, Niehans GA, Gu F, et al. Detection of c-erbB-2 activation in paraffin-embedded tissue by immunohistochemistry. Hum Pathol 1992;23:1141-50.

21 Press MF, Hung G, Godolphin W, et al. Sensitivity of Her2/neu antibodies in archival tissue samples: potential source of error in immunohistochemical studies of oncogene expression. Cancer Res 1994;54:2771-7.

22 Mitchell MS, Press MF. The role of immunohistochemistry and fluorescence in situ hybridization for Her2/neu in assessing the prognosis of breast cancer. Semin Oncol 1999;26:108-16.

23 Press MF, Slamon DJ, Flom KJ, et al. Evaluation of Her-2/neu gene amplification and overexpression: comparison of frequently used assay methods in a molecularly characterized cohort of breast cancer specimens. $J$ Clin Oncol 2002;20:3095-105.

24 Jacobs TW, Gown AM, Yaziii H, et al. Specificity of HercepTest in determining Her2/neu status of breast cancers using the United States Food and Drug Administration-approved scoring system. J Clin Oncol 1999;17:1983-7.

25 Lebeau A, Deimling D, Kaltz C, et al. Her2/neu analysis in archival tissue samples of human breast cancer: comparison of immunohistochemistry and fluorescence in situ hybridization. J Clin Oncol 2001;19:354-63.

26 Pauletti G, Dandekar S, Rong H, et al. Assessment of methods of tissue based detection of the Her2/neu alteration in breast cancer: a direct comparison of fluorescence in situ hybridization and immunohistochemistry. $J$ Clin Oncol 2000;18:3651-64.

27 Roche P, Ingle JN. Increased Her2 expression with U.S. Food and Drug approved antibody. J Clin Oncol 1999;17:434.

28 Maia DM. Immunohistochemical assays for Her2 overexpression. J Clin Oncol 1999:17:1650

29 Bartleft JMS, Going JJ, Mallon EA, et al. Evaluating Her2 amplification and overexpression in breast cancer. $J$ Pathol 2001;195:422-28.

30 Muss $H$, Berry D, Thor A. Lack of interaction of tamoxifen use and erbB2/Her2/neu expression in CALGB 8541: a randomized adjuvant trial of three different doses of cyclophosphamide, doxorubicin and fluorouracil in node positive primary breast cancer. Proc Am Soc Clin Oncol 1999;1999:68A.

31 Hammock L, Lewis M, Phillips C, et al. Strong Her-2/neu protein overexpression by immunohistochemistry often does not predict oncogene amplification by fluorescence in-situ hybridization. Hum Pathol 2003;34:1043-7.

32 Pauletti G, Godolphin W, Press MF, et al. Detection and quantitation of Her$2 /$ neu gene amplification in human breast cancer archival material using fluorescence in situ hybridization. Oncogene 1996;13:63-72.

33 Bartleft J, Mallon E, Cooke T. The clinical evaluation of Her-2 status: which test to use? J Pathol 2003;199:411-17.

34 Ellis IO, Dowsett M, Bartlett J, et al. Recommendations for Her2 testing in the UK. J Clin Pathol 2000;53:890-2.

35 Ellis 1O, Bartlett J, Dowsett M, et al. Updated recommendation for Her2 testing in the UK. J Clin Pathol 2004:57:233-237.

36 Mass RD, Sanders C, Johnson CL, et al. The concordance between the clinical trials assay (CTA) and fluorescence in situ hybridization (FISH) in the Herceptin pivotal trials. Proc Am Soc Clin Oncol 2000;19:75a.

37 Hoang MP, Sahin AA, Ordonez NG, et al. Her2/neu gene amplification compared with Her2/neu protein overexpression and interobserver reproducibility in invasive breast carcinoma. Am J Clin Pathol $2000 ; 113: 852-9$ 